IOS Press

\title{
Use of the cell cycle progression (CCP) score for predicting systemic disease and response to radiation of biochemical recurrence
}

\author{
Michael O. Kocha,*, Jane S. Cho ${ }^{\mathrm{a}}$, Hristos Z. Kaimakliotis ${ }^{\mathrm{a}}$, Liang Cheng ${ }^{\mathrm{b}}$, Zaina Sangale ${ }^{\mathrm{c}}$, \\ Michael Brawer ${ }^{\mathrm{c}}$, William Welbourn ${ }^{\mathrm{c}, 1}$, Julia Reid $^{\mathrm{c}}$ and Steven Stone ${ }^{\mathrm{c}}$ \\ ${ }^{a}$ Department of Urology, Indiana University School of Medicine, Indianapolis, IN, USA \\ ${ }^{\mathrm{b}}$ Department of Pathology and Laboratory Medicine, Indiana University School of Medicine, Indianapolis, IN, \\ USA \\ ${ }^{\mathrm{c}}$ Myriad Genetics, Inc. Salt Lake City, UT, USA
}

\begin{abstract}
.
BACKGROUND: Determining the optimal treatment for biochemical recurrence (BCR) after radical prostatectomy (RP) is challenging.

OBJECTIVE: We evaluated the ability of CCP score (a prognostic RNA expression signature) to discriminate between systemic disease and local recurrence in patients with BCR after RP.

METHODS: Sixty patients with BCR after RP were selected for analysis based on: 1) metastatic disease, 2) non-response to salvage external beam radiotherapy (EBRT), and 3) durable response to salvage EBRT. CCP scores were generated from the RNA expression of 46 genes. Logistic regression assessed the association between CCP score and patient group.

RESULTS: Passing CCP scores were generated for 47 patients with complete clinical and pathologic data. CCP score predicted clinical status when comparing patients with metastatic disease or non-responders to salvage therapy to patients with durable response $(p=0.006)$. CCP score remained significantly predictive of clinical status after accounting for time to BCR, PSA level at BCR, and Gleason score $(p=0.0031)$.

CONCLUSIONS: Elevated CCP score was associated with increased risk of systemic disease, indicating that CCP score may be useful in identifying patients with BCR who are most likely to benefit from salvage radiation therapy.
\end{abstract}

Keywords: Prostate cancer, prostatectomy, cell cycle progression score, biochemical recurrence, salvage radiotherapy

\section{Introduction}

Prostate cancer has a highly variable natural history and accurately assessing the tumor's aggressiveness based on clinical and pathologic features is challenging. After radical prostatectomy, about 15$40 \%$ of patients will experience biochemical recurrence $(\mathrm{BCR})[9,11,13,17]$, which can indicate either

\footnotetext{
${ }^{1}$ Current address: Clinipace Worldwide, Morrisville, NC, USA.

${ }^{*}$ Corresponding author: Michael O. Koch, Department of Urology, Indiana University Simon Cancer Center, 535 Barnhill Drive, Suite 150, Indianapolis, IN 46202, USA. Tel.: +1 317944 7338; Fax: +1 317278 0499; E-mail: miokoch@iupui.edu.
}

metastatic disease or local recurrence. Unfortunately, accurately determining whether a rise in PSA at the time of BCR is due to an isolated prostatic bed recurrence is not possible with the diagnostic tools readily available at this time. Discriminating between these settings is crucial in the selection of appropriate management of BCR patients after prostatectomy. Patients that have metastatic disease are not amenable to curative local therapy. Conversely, salvage radiotherapy for local recurrence is an ideal option as it has been shown to reduce the risk of metastases and improve overall survival of high-risk patients [16].

Conventional imaging such as bone scan, computed tomography (CT) and magnetic resonance imaging

ISSN 1574-0153/16/\$35.00 (c) 2016 - IOS Press and the authors. All rights reserved

This article is published online with Open Access and distributed under the terms of the Creative Commons Attribution Non-Commercial License (CC BY-NC 4.0). 
(MRI) lack the sensitivity to detect specific sites of metastatic disease, especially in patients with PSA less than $10 \mathrm{mg} / \mathrm{ml}$ [7]. Clinical parameters such as time to BCR, PSA doubling time and Gleason score can help to stratify risk of metastatic disease, but their predictive power is limited $[10,19]$. Using an algorithm that combines Gleason score and time to recurrence, men can be stratified into risk groups of $82 \%$ versus $31 \%$ of being free of metastatic disease 5 years after BCR [13]. Moreover, PSA doubling time requires a period of observation, which may result in missing the window of curability with effective salvage radiotherapy.

Variation in the expression of cell cycle progression (CCP) genes has been shown to predict the behavior of a diverse array of cancers including breast, lung, and brain $[12,15,20]$. Furthermore, the CCP score, which is a grading system based on measuring the RNA expression of $31 \mathrm{CCP}$ genes and 15 housekeeping genes, has been associated with prostate cancer outcomes [1,3-5,8].

We evaluated the ability of the CCP score to discriminate between systemic disease (as defined by either metastasis or poor response to salvage radiation) from local recurrence in the setting of BCR after radical prostatectomy (indicated by durable response to salvage radiotherapy) in this pilot study.

\section{Materials and methods}

After Institutional Review Board approval, a retrospective analysis was performed of all patients treated with radical prostatectomy as primary therapy for prostate cancer in our institutional prostate cancer database between 1995 and 2010. We identified all patients for which we had radical prostatectomy paraffin embedded slides that had a BCR and either developed metastatic disease or received external beam salvage radiotherapy with at least 2 years of followup. A cohort of $60 \mathrm{BCR}$ patients was initially identified. BCR was defined as post-operative PSA greater than $0.2 \mathrm{mg} / \mathrm{ml}$ [2] and patients were categorized into one of the following three groups: 1) metastatic disease, 2) non-response to salvage external beam radiotherapy (EBRT) and 3) durable response to salvage EBRT. The metastatic disease cohort included patients with lymph node involvement at the time of surgery or patients who developed evidence of metastasis on bone scan, CT or MRI postoperatively, which were obtained when PSA reached $10 \mathrm{ng} / \mathrm{ml}$ or when they presented with symptoms. Imaging was also obtained in all patients prior to initiation of salvage radiation. Nonresponse patients either had no evidence of biochemical response to salvage EBRT or had two consecutive rises in PSA above $0.1 \mathrm{ng} / \mathrm{ml}$ after nadir in subsequent follow-up. Durable response patients maintained a PSA $<0.1 \mathrm{ng} / \mathrm{ml}$ throughout the study time period with a minimum of two years of follow-up. Patients with a history of adjuvant radiation were excluded.

A board certified pathologist (LC) identified tissue blocks that contained the highest Gleason score which also corresponded to the tumor lesion with the largest diameter. The specimens were then analyzed at Myriad Genetics, Inc. where the selected carcinoma region was macro-dissected, de-paraffinized and RNA extracted according to previously described methods [5]. CCP scores were derived from the expression of 31 previously described CCP genes normalized by the expression of 15 housekeeper genes. Exclusion criteria included insufficient tissue or poor quality RNA resulting in non-passing CCP scores [5].

\subsection{Statistical methods}

A statistical plan was developed prior to data collection and analysis. Logistic regression models were used to evaluate the association of binary outcome with covariates. Effect size was measured by the odds ratio (OR) per one unit change in a given covariate of interest, with a 95\% Wald-type confidence interval (CI). Profile likelihood CIs were calculated for multivariate subset analysis in consideration of the small sample size. Firth's penalized profile likelihood procedure was used for univariate tests of contingency tables with an empty cell. The CCP score was considered continuous and rounded to one decimal place for all analyses. Using the D'Amico risk classification, pathologic Gleason score was considered a 3-level categorical variable for logistic regression modeling, but was converted to integer scoring for calculation of the Pearson correlation coefficient with CCP.

We based all inference on two-sided $p$-values with significance level 0.05. Statistical analysis was carried out at Myriad within the R (version 3.0.1, May 2013; $\mathrm{R}$ Development Core Team) software environment.

\section{Results}

Prostatectomy specimens from 60 patients were sub- 
Table 1

Clinical characteristics among the 47 eligible patients with passing CCP scores

\begin{tabular}{|c|c|c|c|}
\hline \multirow[t]{2}{*}{ Variable } & \multicolumn{3}{|c|}{ Outcome* } \\
\hline & Metastatic disease $N=22$ & Non-responders $N=14$ & Durable response $N=11$ \\
\hline CCP score & $0.6(0.1,1.0)$ & $0.4(-0.2,0.6)$ & $-0.3(-0.6,0.0)$ \\
\hline Age at surgery (years) & $61(56,66)$ & $58(55,62)$ & $58(56,62)$ \\
\hline Pre-surgical PSA (ng/ml) & $10.5(8.0,15.5)$ & $6.5(5.0,9.8)$ & $11.0(5.5,17.0)$ \\
\hline \multicolumn{4}{|l|}{ Pathologic stage } \\
\hline pT2 & $3(14 \%)$ & $4(29 \%)$ & $3(27 \%)$ \\
\hline pT3 & $19(86 \%)$ & $10(71 \%)$ & $8(73 \%)$ \\
\hline \multicolumn{4}{|l|}{ Pathologic Gleason score } \\
\hline$<7$ & $2(9 \%)$ & $2(14 \%)$ & $1(9 \%)$ \\
\hline 7 & $8(36 \%)$ & $7(50 \%)$ & $10(91 \%)$ \\
\hline$>7$ & $12(55 \%)$ & $5(36 \%)$ & $0(0 \%)$ \\
\hline Extra-capsular extension & $18(82 \%)$ & $9(69 \%)^{* *}$ & $8(73 \%)$ \\
\hline Seminal vesicle involvement & $7(32 \%)$ & $3(21 \%)$ & $2(18 \%)$ \\
\hline Positive margins & $14(64 \%)$ & $5(36 \%)$ & $6(55 \%)$ \\
\hline Lymph node invasion & $6(22 \%)$ & $0(0 \%) \dagger$ & $1(11 \%) \dagger \dagger$ \\
\hline Time from surgery to BCR (months) & $12(4,24)$ & $12(1,22)$ & $0(0,21)$ \\
\hline PSA at BCR $(\mathrm{ng} / \mathrm{ml})$ & $0.31(0.20,1.0)$ & $0.14(0.07,0.42)$ & $0.19(0.16,0.26)$ \\
\hline
\end{tabular}

*Summary Measures are Median (IQR) for continuous variables and Counts (Column $\%$ ) for categorical variables. $* * N=13$, due to 1 patient missing status for extra-capsular extension. ${ }^{\dagger} N=13$, due to 1 patient missing data for lymph node invasion. ${ }^{\dagger \dagger} N=9$, due to 2 patients missing data for lymph node invasion.

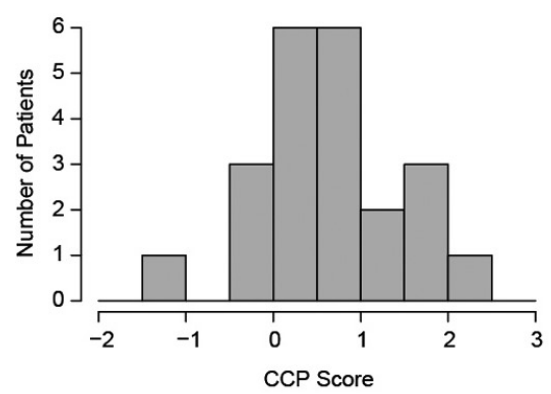

A. Metastatic disease

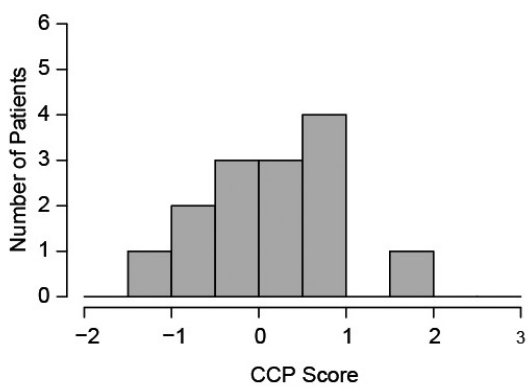

B. EBRT non-responders

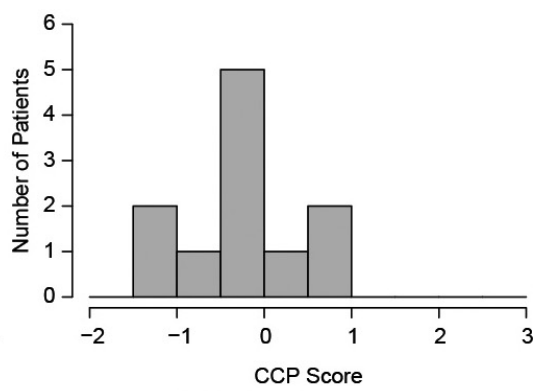

C. EBRT durable response

Fig. 1. Distribution of CCP scores by outcome group: (A) metastatic disease $N=22$; (B) non-responders to EBRT $N=14$; (C) durable response to $\operatorname{EBRT} N=11$.

mitted for CCP gene expression assay. Five patients were excluded from the study for failing to meeting clinical eligibility requirements or because of incomplete clinical data. Sample blocks from 3 patients contained insufficient tumor for assay and 6 patients did not have passing CCP scores. Ultimately, 47 patients were eligible for analysis. Clinical characteristics and demographics of eligible patients are shown in Table 1. Pathologic Gleason scores tended to be higher among patients who had either metastatic disease (55\% had Gleason $>7$ ) or were non-responders to EBRT $(36 \%$ had Gleason > 7) compared to patients with durable response to EBRT. Median follow-up time for the final cohort was 113 months.

The distributions of CCP score appeared similar between patients with confirmed metastatic disease and EBRT non-responders (Fig. 1). All patients in the durable response group had a CCP score less than 1 and while $73 \%$ of the durable responders had a CCP score of less than 0 , only $55 \%$ of the non-durable responders and $18 \%$ of the metastatic disease patients had scores this low.

On univariate logistic regression analysis with binary outcome of metastatic disease and non-responders to salvage EBRT versus the durable response group (Table 2), pathologic Gleason score $(p=0.0073)$ and CCP score $(p=0.006)$ were significantly associated with outcome. The CCP score had an odds ratio (OR) of 3.72 for each one-unit change in the CCP score (95\% CI for OR, 1.29, 10.7). There were no significant interactions between CCP score and any other covariate in predicting outcome ( $p \geq 0.08, p=0.08$ specifically for time from surgery to BCR), and the CCP score was weakly correlated with each of the considered clinical characteristics.

After adjusting for time from surgery to BCR, PSA 
Table 2

Univariate logistic regression models of binary outcome (metastatic disease or non-responders versus durable response)

\begin{tabular}{|c|c|c|c|c|c|}
\hline Variable & $\begin{array}{c}\text { Number of } \\
\text { patients }\end{array}$ & $\begin{array}{l}\text { Odds ratio } \\
(\mathrm{OR})\end{array}$ & $\begin{array}{l}95 \% \text { confidence } \\
\text { interval for OR }\end{array}$ & $P$-value & $\begin{array}{l}\text { Pearson correlation } \\
\text { with CCP score }\end{array}$ \\
\hline CCP score & 47 & 3.72 & $(1.29,10.7)$ & 0.0060 & 1.00 \\
\hline Age at surgery & 47 & 1.03 & $(0.93,1.13)$ & 0.59 & 0.21 \\
\hline Ln $(1+$ pre-surgical PSA $)$ & 47 & 0.90 & $(0.33,2.43)$ & 0.83 & -0.13 \\
\hline \multicolumn{6}{|l|}{ Pathologic stage } \\
\hline $\begin{array}{l}\text { pT2 } \\
\text { pT3 }\end{array}$ & $\begin{array}{l}10 \\
37\end{array}$ & $\begin{array}{c}1.00(\mathrm{ref}) \\
1.55\end{array}$ & $\begin{array}{c}\text { ref } \\
(0.33,7.41)\end{array}$ & 0.59 & 0.26 \\
\hline \multicolumn{6}{|l|}{ Pathologic Gleason scoref } \\
\hline$<7$ & 5 & 1.00 (ref) & ref & & \\
\hline 7 & 25 & 0.49 & $(0.04,3.19)$ & 0.0073 & 0.29 \\
\hline$>7$ & 17 & 11.7 & $(0.53,>100)$ & & \\
\hline Extra-capsular extension* & 35 & 1.27 & $(0.27,5.93)$ & 0.77 & 0.26 \\
\hline Seminal vesicle invasion & 12 & 1.73 & $(0.32,9.45)$ & 0.51 & 0.17 \\
\hline Positive margins & 25 & 0.93 & $(0.24,3.61)$ & 0.92 & 0.20 \\
\hline Lymph node invasion $* * \ddagger$ & 7 & 1.25 & $(0.21,13.2)$ & 0.82 & 0.19 \\
\hline Time from surgery to BCR & 47 & 1.03 & $(0.98,1.08)$ & 0.23 & -0.05 \\
\hline $\mathrm{Ln}(1+\mathrm{PSA}$ at BCR $)$ & 47 & 3.71 & $(0.31,44.0)$ & 0.23 & 0.12 \\
\hline
\end{tabular}

$* 1$ patient missing status for extra-capsular extension. $* * 3$ patients missing status for lymph node invasion. ${ }^{\ddagger} \mathrm{OR}, 95 \% \mathrm{CI}$ for $\mathrm{OR}$, and $p$-value are based upon Firth's penalized profile likelihood.

Table 3

Multivariate logistic regression model of binary outcome (metastatic disease or non-responders versus durable response) with predictors $\mathrm{CCP}$ score, pathologic Gleason score, time to BCR, and PSA at BCR

\begin{tabular}{lccc}
\hline Variable & $\begin{array}{c}\text { Odds } \\
\text { ratio } \\
(\mathrm{OR})\end{array}$ & $\begin{array}{c}95 \% \\
\text { confidence } \\
\text { interval for OR* }\end{array}$ & P-value \\
\hline $\begin{array}{l}\text { CCP score } \\
\text { Pathologic Gleason score }\end{array}$ & 10.4 & $(2.05,90.1)$ & 0.0031 \\
$\quad<7$ & 1.00 (ref) & ref & 0.0016 \\
7 & 0.08 & $(0.00,1.31)$ & \\
$>7$ & $>100$ & $(0.23,>100)$ & \\
Time from surgery to BCR & 1.01 & $(0.96,1.08)$ & 0.70 \\
Ln $(1+$ PSA at BCR) & 2.56 & $(0.16,135)$ & 0.55 \\
\hline
\end{tabular}

*Confidence intervals fit by profile likelihood.

at BCR, and Gleason score in a multivariate logistic regression model (Table 3), the CCP score remained significantly associated with outcome (OR per one unit change in score $=10.4,95 \%$ CI 2.05, 90.1, $p=$ $0.0031)$. When comparing patients with metastatic disease or no response to salvage EBRT to durable response, the CCP score remained significantly associated with outcome, with an OR of 3.64 for a one-unit change in the CCP score (95\% CI for OR, 1.27, 10.5, $p=0.0056$ ).

\section{Discussion}

Novel biomarkers have increasingly become a focus of investigation in prostate cancer as a means of improving prognostic models and determining more appropriate treatment decisions. The CCP score was developed using radical prostatectomy specimens and has been shown to predict not only BCR after radical prostatectomy $[15,20]$, but also BCR after primary EBRT [5] and cancer specific death in conservatively managed cohorts $[4,20]$. These studies described the prognostic utility of CCP scores determined either at disease diagnosis or immediately after radical therapy.

In this study, we find that the CCP score is potentially valuable in the clinical management of patients who experience BCR after prostatectomy. The primary endpoint evaluated was presence of systemic disease, which was broadly defined as patients with either metastasis based on imaging studies, or no response or nondurable response to salvage radiotherapy. Previously, Bishoff et al. conducted a subgroup analysis on the association of CCP scores generated from prostate biopsy specimens to the development of metastatic disease. They found that the association between CCP score and the development of metastasis was much stronger than the association between CCP score and the development of BCR. However, as this was a subgroup analysis, the patient cohort included only 12 patients with metastatic disease [1].

In our pre-planned analysis comparing patients with either metastatic disease or salvage EBRT non-responders to durable responders to salvage, the $\mathrm{CCP}$ score was found to be a significant predictor of outcome $(p=0.0060)$, with the risk of systemic disease increasing 3.72 times for each unit increase of CCP score. CCP score remained highly significant even after taking into account time from surgery and PSA at BCR on multivariate analysis. None of the pa- 
tients with a response to salvage radiotherapy had $\mathrm{CCP}$ scores greater than 1 .

Previously published studies have provided evidence that the association between the CCP score and BCR may be time dependent [4]. In the setting of primary EBRT, Freedland et al. found that the CCP score was strongly associated with early recurrences, but was less strongly associated with late events. The authors theorized that early BCR was probably a sign of micrometastatic disease, whereas later BCR events may be less indicative of truly aggressive disease and less clinically relevant. The data presented here are consistent with that conclusion in that high CCP scores appear to be a harbinger of existing micro-metastatic disease, and identify patients that are unlikely to benefit from localized salvage therapy.

Our results validate previous findings of only a weak correlation between the CCP score and other clinical variables, suggesting that highly proliferative tumors are not easily identifiable based on standard clinicopathologic variables. Because CCP genes have a prominent role in actively replicating cells [18], tumors with high CCP scores may be particularly responsive to future cytotoxic therapies [5].

Other gene expression assays have been utilized to examine post-prostatectomy outcomes and have been shown to predict biochemical free, metastasis free and cancer specific survival [14]. For example, Den et al.compared a genome classifier score between salvage and adjuvant radiation patients and its ability to predict clinical metastasis development [6]. However, these assays have not been shown to predict the likelihood of responding specifically to salvage local radiation after prostatectomy as we are examining in this study.

This study is limited by its retrospective nature and small patient cohort size. Nonetheless, this is the largest population of prostate cancer patients with metastatic disease evaluated for the role of CCP score thus far in this setting. Larger studies are necessary to validate these results and prove that the score adds prognostic information after adjusting for clinical variables.

By delineating the true relationship of CCP score to disease progression and salvage radiotherapy response, an additional diagnostic tool will be available in the treatment of prostate cancer. Ultimately, CCP scores may predict who would benefit from radiotherapy and conversely who will not respond, thereby avoiding the needless side effects and expense from ineffective treatments.

\section{Conclusions}

Among patients treated by radical prostatectomy who develop BCR, CCP score may predict systemic disease and could be used to help guide subsequent therapeutic interventions.

\section{Acknowledgements}

Myriad Genetics, Inc. provided funding for the processing of specimens.

\section{References}

[1] Bishoff JT, Freedland SJ, Gerber L et al., Prognostic utility of the cell cycle progression score generated from biopsy in men treated with prostatectomy. J Urol 192 (2014), 409-414.

[2] Cookson MS, Aus G, Burnett AL et al., Variation in the definition of biochemical recurrence in patients treated for localized prostate cancer: the American Urological Association Prostate Guidelines for Localized Prostate Cancer Update Panel report and recommendations for a standard in the reporting of surgical outcomes. J Urol 177 (2007), 540-545.

[3] Cooperberg MR, Simko JP, Cowan JE et al., Validation of a Cell-Cycle Progression Gene Panel to Improve Risk Stratification in a Contemporary Prostatectomy Cohort. J Clin Oncol 31 (2013), 1428-1434.

[4] Cuzick J, Berney DM, Fisher G et al., Prognostic value of a cell cycle progression signature for prostate cancer death in a conservatively managed needle biopsy cohort. $\mathrm{Br}$ J Cancer 106 (2012), 1095-1099.

[5] Cuzick J, Swanson GP, Fisher G et al., Prognostic value of an RNA expression signature derived from cell cycle proliferation genes in patients with prostate cancer: a retrospective study. Lancet Oncol 12 (2011), 245-255.

[6] Den RB, Yousefi K, Trabulsi EJ et al., Genomic classifier identifies men with adverse pathology after radical prostatectomy who benefit from adjuvant radiation therapy. J Clin Oncol 33 (2015), 944-951.

[7] Dotan ZA, Bianco FJ, Jr., Rabbani F et al., Pattern of prostatespecific antigen (PSA) failure dictates the probability of a positive bone scan in patients with an increasing PSA after radical prostatectomy. J Clin Oncol 23 (2005), 1962-1968.

[8] Freedland SJ, Gerber L, Reid J et al., Prognostic Utility of Cell Cycle Progression Score in Men With Prostate Cancer After Primary External Beam Radiation Therapy. Int J Radiat Oncol Biol Phys 86 (2013), 848-853.

[9] Han M, Partin AW, Pound CR, Epstein JI, Walsh PC. Longterm biochemical disease-free and cancer-specific survival following anatomic radical retropubic prostatectomy, The 15year Johns Hopkins experience. The Urologic Clinics of North America 28 (2001), 555-565.

[10] Joniau S, Briganti A, Gontero P et al., Stratification of highrisk prostate cancer into prognostic categories: a European multi-institutional study. Eur Urol 67 (2015), 157-164.

[11] Kattan MW, Eastham JA, Stapleton AM, Wheeler TM, Scardino PT. A preoperative nomogram for disease recurrence following radical prostatectomy for prostate cancer. $J$ Natl Cancer Inst 90 (1998), 766-771. 
[12] Mosley JD, Keri RA. Cell cycle correlated genes dictate the prognostic power of breast cancer gene lists. BMC Med Genomics 1 (2008), 11.

[13] Pound CR, Partin AW, Eisenberger MA, Chan DW, Pearson JD, Walsh PC. Natural history of progression after PSA elevation following radical prostatectomy. J Am Med Assoc 281 (1999), 1591-1597.

[14] Ross AE, Johnson MH, Yousefi K et al., Tissue-based Genomics Augments Post-prostatectomy Risk Stratification in a Natural History Cohort of Intermediate- and High-Risk Men. Eur Urol 69 (2016), 157-165.

[15] Shedden K, Taylor JMG, Enkemann SA et al., Gene expression-based survival prediction in lung adenocarcinoma: a multi-site, blinded validation study. Nat Med 14 (2008), $822-827$.

[16] Thompson KE, Hernandez J, Canby-Hagino ED, Troyer D,
Thompson IM. Prognostic features in men who died of prostate cancer. J Urol 174 (2005), 553-556.

[17] Ward JF, Moul JW. Rising prostate-specific antigen after primary prostate cancer therapy. Nat Clin Pract Urol 2 (2005), 174-182.

[18] Whitfield ML, Sherlock G, Saldanha AJ et al., Identification of genes periodically expressed in the human cell cycle and their expression in tumors. Mol Biol Cell 13 (2002), 19772000 .

[19] Yossepowitch O, Eggener SE, Serio AM et al., Secondary therapy, metastatic progression, and cancer-specific mortality in men with clinically high-risk prostate cancer treated with radical prostatectomy. Eur Urol 53 (2008), 950-959.

[20] Zhang J, Liu B, Jiang X et al., A systems biology-based gene expression classifier of glioblastoma predicts survival with solid tumors. PloS One 4 (2009), e6274. 